\title{
Meningitis, CTCAE
}

National Cancer Institute

\section{Source}

National Cancer Institute. Meningitis, CT CAE. NCI Thesaurus. Code C143670.

A disorder characterized by acute inflammation of the meninges of the brain and/or spinal cord. 